\title{
Ejercicio físico y suplementación nutricional para el combate de la obesidad sarcopénica en adultos mayores
}

\author{
Héctor Fuentes-Barría ${ }^{*}$ orcid.org/0000-0003-0774-0848 \\ Sebastián Urbano-Cerda' orcid.org/0000-0003-0508-6985 \\ Raúl Aguilera-Eguía² orcid.org/0000-0002-4123-4255 \\ Catalina González-Wong ${ }^{3}$ orcid.org/0000-0003-0360-8567
}

Physical exercise and nutritional supplementation to reduce sarcopenic obesity in older adults

1. Escuela de Ciencias del Deporte y Actividad Física, Facultad de Salud, Universidad Santo Tomás. Chile.

2. Departamento de Salud Pública, Facultad de Medicina, Carrera de Kinesiología. Universidad Católica de la Santísima Concepción. Concepción, Chile.

3. Enfermería, Facultad de Medicina, Universidad del Desarrollo. Santiago, Chile.

\section{Resumen}

Introducción: La obesidad sarcopénica no está claramente definida, pero se entiende como la relación sinérgica entre la pérdida de masa y función muscular, con aumento de masa grasa, propio de la obesidad que se acentúa con la edad. Objetivo: Analizar los efectos del ejercicio físico y suplementación nutricional sobre la obesidad sarcopénica en adultos mayores. Materiales y métodos: Se realizó una búsqueda bibliográfica de artículos publicados entre el 2015 y 2020 en las bases de datos Medline a través de Pubmed, SciELO y Google Scholar utilizando los términos Sarcopenia, Obesity, Frail Elderly, Exercise y Dietary supplements. Los artículos analizados consideraron solo revisiones narrativas o sistemáticas, con o sin metanálisis el efecto de una intervención de ejercicio y/o suplementación nutricional en adultos mayores. Resultados: Se presentan los criterios de definición y relación entre obesidad y sarcopenia abordados desde intervenciones de ejercicios físicos y nutricionales para la prevención y/o tratamiento de obesidad sarcopénica. Conclusiones: Las diferentes estrategias de ejercicio físico y suplementación nutricional, tanto separadas como combinadas, pueden prevenir y/o contrarrestar la obesidad sarcopénica en adultos mayores, puesto que impactan parámetros fundamentales como la composición corporal y el rendimiento físico asociado a la calidad de vida.

Palabras clave: Ejercicio; suplementos dietéticos; obesidad; sarcopenia; anciano frágil. (Fuente: DeCS, Bireme).

\begin{abstract}
Introduction: Although sarcopenic obesity is not clearly defined, it is understood as the synergistic relationship between the loss of muscle mass and function with an increase in fat mass, which characterizes the obesity that increases with age. Objective: To analyze the effects of physical exercise and nutritional supplementation on sarcopenic obesity in older adults. Materials and methods: A bibliographic search of reports published between 2015 and 2020 in the Medline database through Pubmed, SciELO and Google Scholar was conducted, using the terms Sarcopenia, Obesity, Frail Elderly Exercise and Dietary Supplements. The search included narrative and systematic reviews, with or without meta-analyses of the effect of interventions through exercise and/or nutritional supplementation in older adults. Results: The definition criteria and relationship between obesity and sarcopenia were addressed using interventions with physical and nutritional exercises to prevent and/or treat sarcopenic obesity. Conclusions: The different strategies of physical exercise and nutritional supplementation, either individually or in combination, can prevent and/or counteract sarcopenic obesity in older adults, since they affect fundamental parameters such as body composition and the physical performance associated with quality of life.
\end{abstract}

Keywords: Exercise; dietary supplements; obesity; sarcopenia; frail elderly. (Fuente: DeCS, Bireme).

*Autor de correspondencia

Héctor Fuentes-Barría

e-mail: h3ct0r.fuentes.b@gmail.com 


\section{Introducción}

El concepto de obesidad sarcopénica (OS) no está claramente definido, pero se entiende como la relación sinérgica entre la pérdida de masa y función muscular característico de la sarcopenia y el aumento de la masa grasa propio de la obesidad en adultos mayores $(\mathrm{AM})^{(1)}$. Actualmente, se sabe que producto del envejecimiento, tanto la masa como la función muscular disminuyen aproximadamente entre un 3 a $8 \%$ por década, a partir de los 30 a 35 años evidenciando un aumento al pasar los 60 años, mientras que la masa grasa aumenta anualmente entre $0,3 \mathrm{~kg}$ en hombres y 0,4 kg en mujeres(2-4).

La Organización Mundial de la Salud (OMS) ha definido a los AM como toda persona mayor de 60 años, estimando que para el 2020 la población mundial de AM superará a al número de niños, por tanto, los estilo de vida y el nivel de sedentarismo se ha vuelto un importante foco de atención producto de que al menos un $60 \%$ de la población mundial no realiza la actividad física necesaria para obtener beneficios para la salud en Enfermedades Crónicas No Transmisibles (ECNT) como la OS(3,5-7). En este contexto, las recomendaciones de la OMS sugieren realizar $150 \mathrm{~min} / \mathrm{sem}$ de actividad física o un gasto equivalente $>3$ Metabolic equivalent of task (METs por su sigla en inglés) para generar cambios en la composición corporal y parámetros funcionales centrados en el aumento de la masa magra y disminución del tejido adiposo(1,8).

La evidencia actual ha demostrado que programas de actividad física, que incorporen ejercicios de tipo aeróbico, son eficaces en la estimulación de la lipolisis por medio del aumento en la movilización y utilización de los ácidos grasos (AG), mientras que los programas de actividad física que incluyen ejercicios de fuerza muscular son eficaces en la inducción de los mecanismos anabólicos de hipertrofia ${ }^{(9-12) .}$

Recientemente se ha indicado que programas que incorporan ejercicios con cualidades físicas básicas y complementarias en lo que se denomina ejercicios multicomponente (EMC) pueden tener un mayor impacto sobre la salud(3). Información recopilada por el Instituto Australiano del Deporte (AIS por su sigla en inglés), sugiere que el uso del hierro en la dieta otorga un efecto médico real, el cual parece estar relacionado con estados de anemia inflamatoria en OS, además del calcio por su rol sobre la salud ósea y la vitamina D por sus efectos pleiotrópicos (extra óseos) relacionados a la calidad de las fibras musculares tipo II provocando alteraciones en los parámetros de marcha, equilibrio y riesgo de caídas en $A M^{(13)}$. En este sentido, la ingesta dietética recomendada (RDA por su sigla en inglés) para estos elementos oscila entre; $8 \mathrm{mg}$ de hierro, $1200 \mathrm{mg}$ de calcio y 600 a 800 UI/día de vitamina D; sin embargo, estos valores aún son controvertidos debido la heterogeneidad poblacional y los distintos grados basales de vitaminas y minerales $(1,14,15)$. Por estas razones, este estudio se planteó como objetivo analizar los efectos del ejercicio físico y suplementación nutricional sobre la obesidad sarcopénica en adultos mayores.

\section{Materiales y métodos}

Se realizó una revisión narrativa de la literatura mediante búsqueda de artículos científicos en Medline a través de Pubmed, SciELO y Google Scholar. La estrategia de búsqueda se elaboró utilizando los términos Medical Subject Heading Terms (MeSH): Obesity, Sarcopenia, Frail Elderly, Exercise y Dietary Supplements, en forma individual o en conjunto con los operadores booleano " $A N D$ " "OR" con la finalidad de obtener resultados concretos y dirigidos a nuestro objetivo. La búsqueda bibliográfica estuvo limitada a artículos publicados entre el año 2015 a 2020 en idioma inglés y español. Los artículos encontrados por la búsqueda preliminar se analizaron mediante lectura crítica a los resúmenes y/o texto completo, donde fueron evaluados e incluidos según los siguientes criterios (tabla 1).

Tabla 1. Criterios de inclusión y exclusión

\begin{tabular}{lrr}
\hline & \multicolumn{1}{c}{$\begin{array}{c}\text { Criterios de } \\
\text { inclusión }\end{array}$} & $\begin{array}{r}\text { Criterios de } \\
\text { exclusión }\end{array}$ \\
\hline Participantes & $\begin{array}{r}\text { Adultos mayores }(>60 \\
\text { años })\end{array}$ & $\begin{array}{r}\text { Otras poblaciones } \\
(<60 \text { años }) \\
\text { Intervención }\end{array}$ \\
$\begin{array}{lr}\text { Ejercicio físico y/o } \\
\text { suplementación }\end{array}$ & $\begin{array}{r}\text { Otrastervenciones } \\
\text { nutricional }\end{array}$ \\
Comparación & No existe \\
Tipo de & Obesidad sarcopenia & Otras patologías \\
diseño & Revisión narrativa o & Otros diseños \\
& sistemática con o sin & experimentales. \\
& metanálisis & \\
\hline
\end{tabular}


La búsqueda, selección y evaluación de los estudios fue procesada por todos los autores en forma independiente y en caso de desacuerdo, se buscó un consenso según los criterios de inclusión y exclusión. Finalmente, esta revisión referenció 62 artículos, de los cuales se analizaron 5 revisiones que tratan los distintos tipos de ejercicio físico y suplementación nutricional en AM con OS.

Tabla 2. Recomendaciones sobre el ejercicio físico y suplementación nutricional en adultos mayores con obesidad sarcopénica

\begin{tabular}{|c|c|c|c|}
\hline Autor principal & Diseño & Intervención & Resultados principales \\
\hline Goisser, et al.(1) & Narrativa & $\begin{array}{l}\text { Ejercicio físico y/o } \\
\text { suplementación } \\
\text { nutricional }\end{array}$ & $\begin{array}{l}\text { Los ejercicios de tipo aeróbico y de fuerza muscular en combinación } \\
\text { con intervenciones dietéticas, como la suplementación de proteínas } \\
\text { pueden influir la pérdida de peso. }\end{array}$ \\
\hline $\begin{array}{l}\text { Martinez-Amat, et al. } \\
\text { (15) }\end{array}$ & Sistemática & $\begin{array}{l}\text { Ejercicio físico y/o } \\
\text { suplementación } \\
\text { nutricional }\end{array}$ & $\begin{array}{l}\text { Los ejercicios de tipo aeróbico y de fuerza muscular solos o en } \\
\text { combinación con intervenciones dietéticas pueden mejorar los } \\
\text { parámetros de fuerza muscular. }\end{array}$ \\
\hline $\begin{array}{l}\text { Theodorakopoulos, et } \\
\text { al.(16) }\end{array}$ & Sistemática & $\begin{array}{lr}\text { Ejercicio de } & \text { fuerza } \\
\text { muscular } & \text { y/o } \\
\text { suplementación } & \\
\text { nutricional } & \end{array}$ & $\begin{array}{l}\text { La composición corporal no cambió significativamente. Sin embargo, } \\
\text { la intervención de ejercicio mejoró significativamente la fuerza } \\
\text { muscular y la función física. }\end{array}$ \\
\hline Petroni, et al. (17) & Narrativa & $\begin{array}{lr}\text { Ejercicio de } & \text { Fuerza } \\
\text { muscular } & y \\
\text { suplementación } & \\
\text { nutricional } & \end{array}$ & $\begin{array}{l}\text { El entrenamiento de fuerza muscular parece efectivo en la mejora de } \\
\text { la masa muscular, la fuerza y la capacidad funcional, además de la } \\
\text { pérdida de masa grasa, especialmente cuando se combina con dietas } \\
\text { hipocalóricas. Por otro lado, la corrección del déficit de vitamina D } \\
\text { tiene un efecto favorable sobre la masa muscular. }\end{array}$ \\
\hline Trouwborst, et al. (18) & Narrativa & $\begin{array}{l}\text { Ejercicio físico y/o } \\
\text { suplementación } \\
\text { nutricional }\end{array}$ & $\begin{array}{l}\text { Los ejercicios de tipo aeróbico y de fuerza muscular solos o en } \\
\text { combinación con intervenciones dietéticas pueden mejorar la } \\
\text { composición corporal y capacidad funcional. }\end{array}$ \\
\hline
\end{tabular}

\section{Obesidad sarcopénica}

La OS en la actualidad no está claramente definida(1,15). Para el caso del diagnóstico de obesidad organizaciones como la OMS, National Institutes of Health (NIH) y National Cholesterol Education Program Adult Treatment Panel III (ATP III), utilizan como indicador de diagnóstico el índice de masa corporal (IMC) y el perímetro o circunferencia de cintura, estableciendo los siguientes puntos de corte para el diagnóstico de obesidad; IMC entre $30-34,9 \mathrm{~kg} / \mathrm{m}^{2}$ para obesidad leve tipo I, IMC entre $35-39,9 \mathrm{~kg} / \mathrm{m}^{2}$ para obesidad moderada tipo II y IMC $\geq 40 \mathrm{~kg} / \mathrm{m}^{2}$ para obesidad mórbida tipo III, mientras que el diagnóstico según perímetro o circunferencia de cintura se establece en $\geq 102 \mathrm{~cm}$ para hombres $\mathrm{y} \geq 88 \mathrm{~cm}$ para mujeres $(19,20)$. Por otro lado, la literatura busca para el diagnóstico de la sarcopenia evaluar la disminución de los niveles de fuerza muscular, masa muscular y desempeño físico(21).

La OS reporta una prevalencia de entre un 4 a $84 \%$ para hombres y un 4 a $94 \%$ para mujeres,

\section{Resultados}

La tabla 2 resume los aspectos metodológicos de los artículos seleccionados, presentando las 5 revisiones seleccionadas las principales recomendaciones en torno al ejercicio físico y la suplementación nutricional en AM con OS. 


\section{Ejercicio físico}

La práctica de ejercicio tiene un fuerte impacto sobre la calidad de vida relacionada a la capacidad

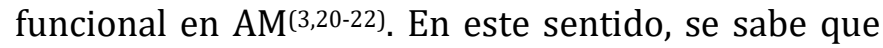
la práctica regular de $150 \mathrm{~min} / \mathrm{sem}$ de ejercicio físico puede producir aumentos de la masa magra y descensos del tejido adiposo generando mejoras en la calidad de vida y disminución del riesgo de padecer ECNT como la OS en $\mathrm{AM}^{(1,2,15,20)}$.

Tradicionalmente se ha planteado, que los ejercicios cardiovasculares o de resistencia aeróbica son eficaces para mejorar la capacidad cardiovascular, mientras que los ejercicios de fuerza muscular son eficaces en la mejora de la función neuromuscular(11,23,24). Actualmente se ha sugerido que programas de EMC pueden tener un mayor impacto en los diversos parámetros de salud ligados a la capacidad funcional en $\mathrm{AM}^{(25-27)}$.

a) Ejercicio aeróbico: Se caracterizan por inducir la movilización, transporte y oxidación de AG originados en la lipolisis, siendo la principal fuerza reguladora del sobrepeso y obesidad debido a su capacidad de elevar hasta 20 veces la tasa metabólica y hasta 100 veces la rotación de ATP muscular del tejido adiposo $y$ subcutáneo(9,11,28,29); además, este tipo de ejercicio también inhibe el estado de lipoinflamación que puede perpetuar la obesidad producto mala utilización de los carbohidratos derivados hacia el hígado para convertirse en depósitos grasos(30).

La evidencia ha propuesto a la alta intensidad (60-85\% del $\mathrm{VO}_{2}$ pico) como una forma efectiva para movilizar AG provenientes de la activación del proceso lipolisis, el cual puede aumentar de dos a tres veces la concentración sérica de AG, mientras que la intensidad moderada (45-65\% $\mathrm{VO}_{2}$ pico) es considerada un factor fundamental en el combate del sobrepeso y obesidad, debido a que en esta zona se encuentra el máximo punto de oxidación de grasas (Fat Max, por su sigla en inglés) considerado por la literatura como un marcador biológico de salud y rendimiento metabólico producto del mayor transporte y oxidación de AG detonantes del catabolismo muscular que puede reducir el riesgo de ECNT por causa de la activación de la cascada de señalización del complejo AMPK que regula las rutas de consumo de ATP impactando la capacidad cardiorrespiratoria, biogénesis mitocondrial, niveles de GLUT4 y resistencia a la insulina entre otros procesos $(9,11,31,32)$. Por esta razón, tradicionalmente la prescripción de este tipo de ejercicio en altas intensidades se fundamenta en la movilización de AG, reducción de las reservas de glucógeno y consumo energético post ejercicio (cascada señalización AMPK), mientras que el ejercicio de moderada intensidad es respaldado por un mayor transporte y la oxidación de AG(11).

b) Ejercicios de fuerza muscular: Estos tipos de ejercicios tradicionalmente se han prescrito para el tratamiento y/o prevención de la OS debido a que estimulan de manera eficaz la fuerza e hipertrofia muscular, además de mejorar parámetros de capacidad funcional como; velocidad de marcha, equilibrio (estático y dinámico) y riesgo de accidentes por caídas en AM; sin embargo, su prescripción siempre debe considerar los principios del entrenamiento deportivo como la individualidad, especificidad y progresión de la carga de trabajo que puede impactar los parámetros ligados a la salud(24,33).

El desarrollo de la fuerza máxima en pacientes obesos generalmente es mayor en comparación a personas sanas, puesto que el tejido adiposo actual como estímulo de sobrecarga, sin embargo, al normalizar la fuerza máxima en función de la masa corporal generalmente los pacientes obesos son más débiles en comparación a un normopeso(34), mientras que en pacientes con sarcopenia el desarrollo de la fuerza máxima es uno de los criterios de diagnóstico clínico evaluado mediante indicadores como las relaciones; fuerza/IMC $<1,00$, masa magra apendicular/IMC $<0,789$ y fuerza máxima de agarre $<26 \mathrm{~kg}(12)$.

El ejercicio de fuerza muscular modifica la composición corporal mediante la activación de la cascada señalización del complejo rapamicina (mTOR), esta vía al igual que el AMPK modula los procesos de anabolismo y catabolismo celular dependiendo de la intensidad del ejercicio(35). En este contexto, los ejercicios catalogados como de fuerza/velocidad $(50 \%$ repetición máxima) pueden generar cambios positivos a corto plazo en los parámetros de fuerza muscular y capacidad funcional, no así en la composición 
corporal, puesto que el objetivo del entrenamiento a corto plazo siempre será mejorar la capacidad funcional asociada al riesgo de invalidez y la adherencia a programas de actividad física, mientras que los ejercicios de fuerza/ resistencia ( $<50 \%$ repetición máxima) son los más efectivos en la mejora parámetros de fuerza muscular, capacidad funcional $y$ composición corporal, puesto que suponen un efectos a mediano y largo plazo(36-38).

c) Ejercicio multicomponente (EMC): Se definen como una combinación de las distintas cualidades físicas básicas (capacidad aeróbica, fuerza muscular y velocidad) y complementarias (amplitud de movimiento, coordinación y equilibrio) generalmente prescritos en base a la valoración de parámetros funcionales como; velocidad de marcha, equilibrio y agilidad los cuales son claves en la prevención y diagnóstico de ECNT como la OS en AM(3,28-30).

\section{Suplementación nutricional}

Corresponden a la ingesta intencionada de un alimento, componente alimenticio, nutriente o compuesto no alimenticio en forma adicional a la dieta habitual, con el objetivo de lograr un beneficio específico de salud y/o rendimiento físico(39). Actualmente existen un sin número de suplementos nutricionales en el mercado los cuales muchas veces son comercializados a pesar de no tener atributos sobre la salud comprobados, motivo por el cual instituciones como el AIS han debido revisar y clasificar los suplementos nutricionales según el nivel de evidencia científica con relación a sus atributos, concluyendo que aquellos suplementos con mejor evidencia científica para uso médico (grupo A) en el combate de patologías como la OS son los siguientes(14):

a) Hierro: Este mineral tradicionalmente se ha utilizado para el tratamiento y/o prevención de estados deficitarios de hemoglobina $(<12 \mathrm{~g} / \mathrm{dl}$ para hombres y $<13 \mathrm{~g} /$ dl para mujeres) o anemia en AM con OS, puesto que el estado de lipoinflamación puede sobre estimular las rutas de señalización de la hepcidina, degradando la ferroportina e inhibiendo la absorción del hierro por parte de los enterocitos estimulando la captación o secuestro de este por parte de los macrófagos, siendo este mineral también fundamental en la práctica de ejercicio de resistencia aeróbica, ya que este altera el metabolismo pudiendo inducir disminuciones en los niveles de hierro producto de la disminución del número de eritrocitos ${ }^{(40,41)}$.

La evidencia ha planteado que una RDA óptima en torno a los $14 \mathrm{mg}$ puede aumentar en 0,35 $\mathrm{g} / \mathrm{dl}$ los niveles de hemoglobina luego de un periodo de 4 a 6 semanas en población de AM, mientras que dosis $>15-20 \mathrm{mg} /$ día pueden inhibir las vías de absorción de este elemento(42,43). En este contexto, el hierro mejora los niveles de hemoglobina, además del transporte y producción energética en ejercicios de tipo aeróbicos, no obstante, este también podría relacionarse a desórdenes neurodegenerativos sobre parámetros de capacidad funcional como el riesgo de caídas e

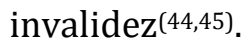

b) Calcio: Este macronutriente es esencial en la dieta ya que cumple un rol fundamental en la mantención y promoción de la salud ósea en AM, además de regular los procesos de contracción muscular, reacciones enzimáticas y metabolismo celular (mitocondrial, retículo endoplásmico, apoptosis, autofagia e inflamación), puesto que altos niveles séricos de este macronutriente se han relacionado a la reducción de la expresión de la sintasa de AG y el manejo indirecto del balance energético y adiposidad, sin embargo, aún no existe evidencia contundente que respalde esta relación(46-51). En cuanto al ejercicio físico la evidencia ha observado que la duración e intensidad de carga están condicionadas por la disponibilidad de este elemento en el retículo sarcoplasmático que a su vez está modulado por el nivel de fatiga y agotamiento del glucógeno muscular, además de los niveles séricos de vitamina $\mathrm{D}(50,52-54)$.

Por esta razón, la suplementación de calcio comúnmente se realiza en conjunto con la vitamina $\mathrm{D}$ con el objetivo de promover la salud ósea y prevenir patologías como la osteopenia solo reportando efectos adversos relacionados al mayor riesgo de desarrollar cálculos renales y mortalidad cardiovascular en dosis superiores a la RDA (1000 a 1200 UI/día) (50,53,54).

c) Vitamina D: Como se acaba de exponer a esta vitamina se le atribuyen efectos sobre la 
absorción de calcio y fosfato, además de posibles efectos protectores sobre las ECNT(54-56). En la literatura aún no existe un consenso entre las clasificaciones y niveles de Vitamina D, sin embargo, la evidencia ha reportado que niveles $<20 \mathrm{ng} / \mathrm{ml}$ se asocian con OS y la pérdida de fuerza y masa muscular producto de la disminución en la calidad biológica de las fibras musculares tipo II, las cuales son las primeras en reclutarse en situaciones de riesgo como las caídas en $\mathrm{AM}$, mientras que, niveles $\geq 50 \mathrm{ng} / \mathrm{ml}$ se han relacionado con alteraciones del ritmo cardiaco, estados de confusión y desorientación(57-59). Organizaciones como el Instituto de Medicina de Estados Unidos utilizan una RDA >800 UI/día para AM sobre 70 años, mientras otras como la Sociedad de Endocrinología plantean una dosis cercana a las 4000 UI/día para estados de obesidad(59).

\section{Discusión}

A pesar de que no existe una definición universalmente aceptada para la OS, ésta se entiende en forma global como la combinación de diferentes mecanismos comunes entre la obesidad y sarcopenia que implican riesgos para la salud, tales como los desórdenes endocrinos y factores inflamatorios causados por el incremento de las conductas sedentarias y la malnutrición que promueven los estilos poco saludables(1,15,23). No obstante, estas condiciones se pueden prevenir y/o tratar mediante el uso de diversas intervenciones centradas en el ejercicio físico y suplementación nutricional.

Las recomendaciones tradicionales en torno al ejercicio plantean priorizar el desarrollo de la capacidad funcional a corto plazo mediante la utilización de ejercicios de fuerza muscular que busquen mejorar la composición corporal producto de la hipertrofia inducida por las adaptaciones ligadas a la intensidad de la carga(60), mientras que a mediano y largo plazo se sugiere incorporar el uso de ejercicios de tipo aeróbicos fundamentados en la mejora de la composición corporal producto de la movilización y transporte de AG(11). En este contexto, se debe considerar que las recomendaciones actuales sugieren la utilización de EMC que incorpore aspectos como el equilibrio, coordinación, flexibilidad, capacidad aeróbica de intensidad moderada y fuerza muscular con énfasis en la resistencia y velocidad, puesto que estas combinaciones estimulan un efecto mucho más potente sobre la mejora de parámetros físicos y biológicos en comparación a programas de ejercicios tradicionales unidireccionales, reportado mejoras sobre el perfil lipídico y composición corporal, además de parámetros de rendimiento físico como la capacidad aeróbica, fuerza muscular, equilibrio, flexibilidad y función cognitiva los cuales impactan en la capacidad funcional y calidad de vida de los $\operatorname{AM}(30,60-62)$.

En cuanto a la prescripción de suplementación nutricional, esta debe considerar la existencia de deficiencias nutricionales, puesto que estados séricos deficientes pueden generar diversos efectos perjudiciales sobre la salud de AM. En este contexto, la evidencia científica sintetizada por el AIS recomienda solo suplementar hierro, calcio $\mathrm{y}$ vitamina $\mathrm{D}$ para prevenir o tratar problemas clínicos, incluidas las deficiencias de nutrientes diagnosticadas en ECNT como la OS(13). Entre los suplementos más ampliamente utilizados en AM con OS solo destacan la vitamina $\mathrm{D}$ y el calcio a pesar de que solo poseen un efecto comprobado sobre la salud musculoesquelética, siendo los efectos pleiotrópicos aún controvertidos( ${ }^{(50,59)}$. En este sentido, la RDA de vitamina D suelen estar entre 600 a $800 \mathrm{UI} /$ día para adultos entre sobre 50 años sin riesgo de deficiencia y en entre 1500 a 2000 UI/día para adultos con riesgo de deficiencia, mientras que para el calcio se sitúa en torno a las 1000 a 1200 UI/día ${ }^{(50,59) .}$

Este artículo propone profundizar en futuras investigaciones la definición del concepto OS, con el objetivo de proporcionar guías que permitan un mejor diagnóstico, prevención y tratamiento en los AM. Estas recomendaciones de ejercicio físico y suplementación nutricional son importantes en cualquier etapa de la vida en particular en condiciones de estilos de vida poco saludables como los que generalmente presentan los AM.

\section{Conclusiones}

Las diferentes estrategias de ejercicio físico y suplementación nutricional, tanto separadas como combinadas, pueden prevenir y/o contrarrestar la OS en $\mathrm{AM}$, puesto que impactan parámetros fundamentales como la composición corporal y el rendimiento físico asociado a la calidad de vida.

\section{Conflicto de intereses}

Ninguno declarado por los autores. 


\section{Referencias}

1. Goisser S, Kemmler W, Porzel S, Volkert D, Sieber CC, Bollheimer LC, et al. Sarcopenic obesity and complex interventions with nutrition and exercise in communitydwelling older persons - A narrative review. Clin Interv Aging. 2015;10:1267-82. doi: 10.2147/CIA.S82454.

2. Zeng P, Han Y, Pang J, Wu S, Gong H, Zhu J, et al. Sarcopeniarelated features and factors associated with lower muscle strength and physical performance in older Chinese: A cross sectional study Physical functioning, physical health and activity. BMC Geriatr. 2016;16(1):45. doi: 10.1186/s12877016-0220-7.

3. Chalapud Narváez LM, Escobar Almario AE. Actividad física para mejorar fuerza y equilibrio en el adulto mayor. Univ y Salud. 2017;19(1):94-101. doi: 10.22267/rus.171901.73

4. Gutiérrez Cortés W, Martínez Fernández F, Olaya Sanmiguel L. Sarcopenia, una patología nueva que impacta a la vejez. Rev ACE. 2018;5(1):28-36. Disponible en: http://revistaendocrino.org/index.php/rcedm/article/view /339

5. Varela Pinedo LF. Salud y calidad de vida en el adulto mayor. Rev Peru Med Exp Salud Publica. 2016;33(2):199201. doi: 10.17843/rpmesp.2016.332.2196

6. OMS. Envejecimiento y ciclo de vida [Internet]. [citado 2019 Sep 25]. Disponible en: https://www.who.int/features/factfiles/ageing/ageing_fact s/es/

7. Romero Blanco C, Artiga González MJ, Cabanillas Cruz E, Casajús Mallén JA, Ara Royo I, Aznar Laín S. Obesidad sarcopénica en mujeres mayores: Influencia del polimorfismo I/D de la enzima convertidora de angiotensina. Nutr Hosp. 2017;34(5):1099-104. doi: $10.20960 /$ nh.913

8. OMS. Recomendaciones mundiales sobre la actividad física para la salud [Internet]. [citado 2019 Sep 25]. Disponible en:

https://www.who.int/dietphysicalactivity/factsheet_recom mendations/es/

9. Noland RC. Exercise and Regulation of Lipid Metabolism. Progress in Molecular Biology and Translational Science. 2015;135:39-74. doi: 10.1016/bs.pmbts.2015.06.017

10. Purdom T, Kravitz L, Dokladny K, Mermier C. Understanding the factors that effect maximal fat oxidation. J Int Soc Sports Nutr. 2018;15(1):3. doi: 10.1186/s12970-018-0207-1

11. Espinoza-Salinas A, González-Jurado J, Molina-Sotomayor E, Fuentes-Barria H, Farias-Valenzuela C, Arenas Sanchez G. Mobilization, transport and oxidation of fatty acids: physiological mechanisms associated with weight loss. J Sport Heal Res. 2020;12(13):303-12. Disponible en https://recyt.fecyt.es/index.php/JSHR/article/view/81308

12. Solano García W, Carazo Vargas P. Intervenciones con ejercicio contra resistencia en la persona adulta mayor diagnosticada con sarcopenia. Una revisión sistemática. Pensar en Mov. 2018;16(1):30000. doi:10.15517/pensarmov.v16i1.3000.

13. Supplements | Australian Institute of Sport [Internet]. [citado 2019 Sep 25]. Disponible en: https://www.ais.gov.au/nutrition/supplements\#group_a

14. NIH. Fact Sheets on Individual Dietary Supplement Ingredients [Internet]. [citado 2019 Sep 25]. Disponible en: https://ods.od.nih.gov/HealthInformation/RecursosEnEspa nol.aspx
15. Martínez-Amat A, Aibar-Almazán A, Fábrega-Cuadros R, Cruz-Díaz D, Jiménez-García JD, Pérez-López FR, et al. Exercise alone or combined with dietary supplements for sarcopenic obesity in community-dwelling older people: a systematic review of randomized controlled trials. Maturitas. 2018;110:92-103. doi: 10.1016/j.maturitas.2018.02.005.

16. Theodorakopoulos C, Jones J, Bannerman E, Greig CA. Effectiveness of nutritional and exercise interventions to improve body composition and muscle strength or function in sarcopenic obese older adults: A systematic review. Nutr Res. 2017;43:3-15. doi: 10.1016/j.nutres.2017.05.002.

17. Petroni ML, Caletti MT, Grave RD, Bazzocchi A, Aparisi Gómez MP, Marchesini G. Prevention and treatment of sarcopenic obesity in women. Nutrients. 2019;11(6) pii: E1302. doi: 10.3390/nu11061302.

18. Trouwborst I, Verreijen A, Memelink R, Massanet P, Boirie $\mathrm{Y}$, Weijs $\mathrm{P}$, et al. Exercise and nutrition strategies to counteract sarcopenic obesity. Nutrients. 2018;10(5) pii: E605. doi: 10.3390/nu10050605.

19. Lecube A, Monereo S, Rubio MÁ, Martínez-de-Icaya P, Martí A, Salvador J, et al. Prevención, diagnóstico y tratamiento de la obesidad. Posicionamiento de la Sociedad Española para el Estudio de la Obesidad de 2016. Endocrinol Diabetes Nutr. 2017;64:15-22. doi: 10.1016/j.endonu.2016.07.002.

20. Castro-Martínez MG, Liceaga-Craviotto G, AlexandersonRosas G, Bolado-García V, d'Hyver-Wiechers C, DuránSalgado $M$, et al. Consenso de expertos en prevención, diagnóstico y tratamiento de la obesidad en el adulto mayor y en casos especiales. Med Int Méx. 2016;32(1):58-88. Disponible en https://www.medigraphic.com/cgibin/new/resumen.cgi?IDARTICULO $=64342$

21. Hernández-Rodríguez J, Arnold Domínguez Y. Principales elementos a tener en cuenta para el correcto diagnóstico de la sarcopenia. Medisur. 2019;17(1):112-25. Disponible en http://scielo.sld.cu/scielo.php?script=sci_arttext\&pid=S172 7-897X2019000100112\&lng=es\&nrm=iso\&tlng=es

22. Cruz-Jentoft AJ, Bahat G, Bauer J, Boirie Y, Bruyère 0 , Cederholm T, et al. Sarcopenia: Revised European consensus on definition and diagnosis. Age Ageing. 2019;48(1):16-31. doi: 10.1093/ageing/afy169.

23. Kalinkovich A, Livshits G. Sarcopenic obesity or obese sarcopenia: A cross talk between age-associated adipose tissue and skeletal muscle inflammation as a main mechanism of the pathogenesis. Ageing Res Rev. 2017;35:200-21. doi: 10.1016/j.arr.2016.09.008.

24. Steffl M, Bohannon RW, Sontakova L, Tufano JJ, Shiells K, Holmerova I. Relationship between sarcopenia and physical activity in older people: a systematic review and metaanalysis. Clin Interv Aging. 2017;12:835-45. doi: 10.2147/CIA.S132940.

25. Sherrington C, Michaleff ZA, Fairhall N, Paul SS, Tiedemann A, Whitney J, et al. Exercise to prevent falls in older adults: an updated systematic review and meta-analysis. Br J Sports Med. 2017;51(24):1749-57. doi: 10.1136/bjsports-2016096547

26. Romero SA, Minson CT, Halliwill XR. The cardiovascular system after exercise. J Appl Physiol. 2017;122(4):925-32. doi: 10.1152/japplphysiol.00802.2016.

27. Papa EV, Dong X, Hassan M. Resistance training for activity limitations in older adults with skeletal muscle function deficits: a systematic review. Clin Interv Aging. 2017;12:955-61. doi: 10.2147/CIA.S104674. 
28. Liberman K, Forti LN, Beyer I, Bautmans I. The effects of exercise on muscle strength, body composition, physical functioning and the inflammatory profile of older adults: a systematic review. Curr Opin Clin Nutr Metab Care. 2017;20(1):30-53. doi: 10.1097/MC0.0000000000000335.

29. Low DC, Walsh GS, Arkesteijn M. Effectiveness of exercise interventions to improve postural control in older adults: a systematic review and meta-analyses of centre of pressure measurements. Sport Med. 2017;47(1):101-12. doi: 10.1007/s40279-016-0559-0.

30. Karssemeijer EGA, Aaronson JA, Bossers WJ, Smits T, Olde Rikkert MGM, Kessels RPC. Positive effects of combined cognitive and physical exercise training on cognitive function in older adults with mild cognitive impairment or dementia: a meta-analysis. Ageing Res Rev. 2017;40:75-83. doi: 10.1016/j.arr.2017.09.003.

31. Booth FW, Ruegsegger GN, Toedebusch RG, Yan Z. Endurance exercise and the regulation of skeletal muscle metabolism. Prog Mol Biol Transl Sci. 2015;135:129-51. doi: 10.1016/bs.pmbts.2015.07.016.

32. González-Ruiz K, Ramírez-Vélez R, Correa-Bautista JE, Peterson MD, García-Hermoso A. The effects of exercise on abdominal fat and liver enzymes in pediatric obesity: a systematic review and meta-analysis. Child Obes. 2017;13(4):272-82. doi: 10.1089/chi.2017.0027

33. Izaola 0 , de Luis $D$, Sajoux I, Domingo JC, Vidal M. Inflamación y obesidad (Lipoinflamación). Nutr Hosp. 2015;31(6):2352-8. doi: 10.3305/nh.2015.31.6.8829.

34. Amaro-Gahete FJ, Sanchez-Delgado G, Jurado-Fasoli L, Dela-0 A, Castillo MJ, Helge JW, et al. Assessment of maximal fat oxidation during exercise: a systematic review. Scand J Med Sci Sport. 2019;29(7):910-21. doi: 10.1111/sms.13424.

35. Burgos Jara C, Henríquez-Olguín C, Ramírez-Campillo R, Matsudo SM, Cerda-Kohler H, Mahecha Matsudo S. Exercise as a tool to reduce body weight. Rev Med Chil. 2017;145(6):765-74. doi: 10.4067/s003498872017000600765

36. Giallauria F, Cittadini A, Smart NA, Vigorito C. Resistance training and sarcopenia. Monaldi Arch Chest Dis - Card Ser. 2015;84(1-2):738. doi: 10.4081/monaldi.2015.738.

37. Tomlinson DJ, Erskine RM, Morse CI, Winwood K, Onambélé-Pearson G. The impact of obesity on skeletal muscle strength and structure through adolescence to old age. Biogerontology. 2016;17(3):467-83. doi: 10.1007/s10522-015-9626-4.

38. Haissaguerre M, Cota D. Role of the mTOR pathway in the central regulation of energy balance. Biol Aujourdhui. 2015;209(4):295-307. doi: 10.1051/jbio/2016009.

39. Maughan RJ, Burke LM, Dvorak J, Larson-Meyer DE, Peeling P, Phillips SM, et al. IOC consensus statement: dietary supplements and the high-performance athlete. Br J Sports Med. 2018;52(7):439-55. doi: 10.1136/bjsports-2018099027.

40. Joosten E. Iron deficiency anemia in older adults: A review. Geriatr Gerontol Int. 2018;18(3):373-9. doi: 10.1111/ggi.13194.

41. Salazar-Lugo R. Metabolismo del hierro, inflamación y obesidad. Saber. 2015;27(1):5-16. Disponible en http://ve.scielo.org/scielo.php?script=sci_arttext\&pid=S13 15-01622015000100002

42. DeLoughery TG. Iron Deficiency Anemia. Med Clin North Am. 2017;101(2):319-32. doi: 10.1016/j.mcna.2016.09.004.
43. Tay HS, Soiza RL. Systematic review and meta-analysis: what is the evidence for oral iron supplementation in treating anaemia in elderly people? Drugs Aging. 2015;32(2):149-58. doi: 10.1007/s40266-015-0241-5.

44. Alaunyte I, Stojceska V, Plunkett A. Iron and the female athlete: a review of dietary treatment methods for improving iron status and exercise performance. J Int Soc Sports Nutr. 2015;12(38):1-7 doi: 10.1186/s12970-0150099-2

45. Apostolakis S, Kypraiou AM. Iron in neurodegenerative disorders: being in the wrong place at the wrong time? Rev Neurosci. 2017;28(8):893-911. doi: 10.1515/revneuro2017-0020.

46. Reid IR, Bristow SM, Bolland MJ. Calcium supplements: benefits and risks. J Intern Med. 2015;278(4):354-68. doi: 10.1111/joim.12394.

47. Dasarathy J, Labrador H. Bone health in women. Prim Care Clin Off Pract. 2018;45(4):643-57. doi: 10.1016/j.pop.2018.07.011.

48. Tu MK, Levin JB, Hamilton AM, Borodinsky LN. Calcium signaling in skeletal muscle development, maintenance and regeneration. Cell Calcium. 2016;59(2-3):91-7. doi: 10.1016/j.ceca.2016.02.005.

49. Ahn C, Kang JH, Jeung EB. Calcium homeostasis in diabetes mellitus. J Vet Sci. 2017;18(3):261-6. doi: 10.4142/jvs.2017.18.3.261.

50. Aguilera Eguía R, Jorquera Pino PJ, Jaqueline Salgado C, Flores C. Suplementación de calcio para la disminución de peso en personas con obesidad; un overview de revisiones sistemáticas. Nutr Hosp. 2016;33(2):464-71. doi: 10.20960/nh.524

51. Szymański J, Janikiewicz J, Michalska B, Patalas-Krawczyk P, Perrone M, Ziółkowski W, et al. Interaction of mitochondria with the endoplasmic reticulum and plasma membrane in calcium homeostasis, lipid trafficking and mitochondrial structure. Int J Mol Sci. 2017;18(7):pii: E1576. doi: 10.3390/ijms18071576

52. Cheng AJ, Place N, Westerblad H. Molecular basis for exercise-induced fatigue: the importance of strictly controlled cellular Ca 2+ handling. Cold Spring Harb Perspect Med. 2018;8(2):pii: a029710. doi: 10.1101/cshperspect.a029710.

53. Martinez de Victoria E. El calcio, esencial para la salud. Nutr Hosp. 2016;33(4):26-31. doi:10.20960/nh.341

54. Aivar Blanch M. La vitamina D y el calcio en la prevención de fracturas. Enferm Clin. 2015;25(3):146-8. doi: 10.1016/j.enfcli.2015.02.004

55. Liu J, Dong Y, Lu C, Wang Y, Peng L, Jiang M, et al. Metaanalysis of the correlation between vitamin $\mathrm{D}$ and lung cancer risk and outcomes. Oncotarget. 2017;8(46):8104051. doi: 10.18632/oncotarget.18766

56. Qi D, Nie X, Wu S, Cai J. Vitamin D and hypertension: prospective study and meta-analysis. PLoS One. 2017;12(3):e0174298. doi: 10.1371/journal.pone.0174298.

57. Annweiler C, Henni S, Walrand S, Montero-Odasso M, Duque G, Duval GT. Vitamin D and walking speed in older adults: systematic review and meta-analysis. Maturitas. 2017;106:8-25. doi: 10.1016/j.maturitas.2017.07.012

58. Lappe JM, Binkley N. Vitamin D and sarcopenia/falls. J Clin Densitom. 2015;18(4):478-82. doi: 10.1016/j.jocd.2015.04.015.

59. Fuentes Barria H, Aguilera Eguía R, González Wong C. El rol de la vitamina $\mathrm{D}$ en la prevención de caídas en sujetos con 
sarcopenia. Rev Chil Nutr. 2018;45(3):279-84. doi: 10.4067/S0717-75182018000400279.

60. Bouaziz W, Lang PO, Schmitt E, Kaltenbach G, Geny B, Vogel T. Health benefits of multicomponent training programmes in seniors: a systematic review. Int J Clin Pract. 2016;70(7):520-36. doi: 10.1111/ijcp.12822.

61. de Asteasu MLS, Martínez-Velilla N, Zambom-Ferraresi F, Casas-Herrero Á, Izquierdo M. Role of physical exercise on cognitive function in healthy older adults: A systematic review of randomized clinical trials. Ageing Res Rev. 2017;37:117-34. doi: 10.1016/j.arr.2017.05.007.

62. Booth V, Hood V, Kearney F. Interventions incorporating physical and cognitive elements to reduce falls risk in cognitively impaired older adults: a systematic review. JBI database Syst Rev Implement reports. 2016;14(5):110-35. doi:

10.11124/JBISRIR-2016-002499 